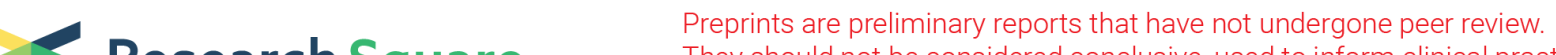 Research Square They should not be considered conclusive, used to inform clinical practice, or referenced by the media as validated information.
}

\section{Septic Patients Benefited from Positive Microbial Culture in Mortality: A Propensity Score Matching (PSM) of the Chinese Epidemiological Study of Sepsis (CHESS) Study}

\section{Wei Chang}

Jiangsu Provincial Key Laboratory of Critical Care Medicine,Department of Critical Care Medicine, Zhongda Hospital, Southeast University

Jianfeng Xie

Southeast University Zhongda Hospital

\section{Qin Sun}

Southeast University Zhongda Hospital

\section{Fei Peng}

Southeast University Zhongda Hospital

Qing Li

Southeast University Zhongda Hospital

\section{Ming Xue}

Southeast University Zhongda Hospital

\section{Yuan Zhao}

Southeast University Zhongda Hospital

\section{Haibo Qiu}

Southeast University Zhongda Hospital

Yi Yang ( $\nabla$ yiyiyang2004@163.com )

Nanjing Zhongda Hospital, Southeast University https://orcid.org/0000-0003-1661-3568

\section{Research}

Keywords: sepsis, microbial culture, mortality

Posted Date: August 12th, 2020

DOl: https://doi.org/10.21203/rs.3.rs-56031/v1

License: (c) (i) This work is licensed under a Creative Commons Attribution 4.0 International License. Read Full License 


\section{Abstract}

Background We aimed to investigate the characteristics and outcomes in septic patients with positive cultures compared with negative cultures.

Methods This was a sub-analysis of a cross-section survey study including 44 hospitals in mainland China from December 1, 2015 to January, 31,2016. All septic patients diagnosed according to sepsis-1 criteria admitted to participating ICU, with cultures from any suspected sites of infection obtained at admission. Demographic information, patient source, clinical feature, laboratory findings and severity of sepsis were comprehensively collected for comparison the characteristics between culture positive and negative patients. Univariable and multivariable regression were used to find the factors associated with positive culture and propensity score matching (PSM) was performed to compare the mortality.

Results A total of 1919 patients were enrolled in this study with 962 (50.1\%) positive-culture and 975 (49.9\%) negative-culture. Positive culture was associated with older age, male gender, transferred patients, comorbidities including coronary heart decease and solid tumor, prolonged priori ICU stay, hospital-acquired infection, pneumonia and bloodstream infection, also with higher body temperature, heart rate, procalcitonin levels and with higher APACHE II and SOFA score at admission. Negative culture was associated with prior antibiotic exposure and community-acquired infections. Patients with positive culture had an insignificantly higher mortality ( $37.5 \%$ vs. $34.5, P=0.165)$. However, PSM revealed decreased mortality in patients with positive culture (38.2\% vs. $48.2 \%, P=0.012)$, and a significantly higher proportion of patients received targeted antibiotic treatment in survivors (37.0\% vs. $46.2 \%, P=$ $0.031)$.

Conclusions A myriad of factors may affect the results of culture. Septic patients were likely to benefit from positive cultures in mortality possibly due to the higher proportion to receive targeted antibiotic treatment, and obtaining positive culture as possible should be strongly encouraged.

\section{Backgrounds}

Sepsis is defined as life-threatening organ dysfunction due to dysregulated immune response to microorganism invasion ${ }^{[1]}$. In spite of advances in recent years, the mortality of sepsis remains as high as 30$60 \%{ }^{[2]}$, which is a heavy burden to the healthcare system and causes more than five million death each year ${ }^{[3]}$.

Early obtaining of appropriate routine microbiologic cultures both from blood and suspected infection sites is strongly recommended in the Surviving Sepsis Campaign International Guidelines $(2016)^{[4]}$. This is of great importance since detection of micro-organisms which possibly caused sepsis would be of great help in targeted antibiotics therapy and de-escalation, which is associated with improved mortality ${ }^{[5]}$. 
However, culture-negative is common among septic patients which ranged from approximately $30-70 \%$ according to the previous studies under different settings, and the characteristics of the patients with positive culture have been sparsely investigated and poorly identified.

Although early empirical antibiotics was administered discriminatively regardless of the culture results as the initial sepsis management recommended in the SSC International Guidelines (2016), the difference in mortality between septic patients with positive and negative culture from previous studies remains inconsistent ${ }^{[6-8]}$, which needs further validations.

In this study, we aimed to comprehensively collect the demographic characteristics, comorbidities, clinical features, laboratory findings and initial treatments at admittance, to explore the potential factors associated with culture positivity in septic patients. Furthermore, we aimed to investigate the association between positive culture and the patient's outcomes.

\section{Materials And Methods Design and settings}

This was a sub-analysis of Chinese Epidemiological Study of Sepsis (CHESS) study ${ }^{[9]}$, which was a multicenter prospective cross-sectional survey performed in mainland China involving 44 hospitals, commenced on December 1, 2015 and concluded on January 31, 2016. Patients with suspected or confirmed sepsis admitted to intensive care unit (ICU) and with blood and/or any other samples from suspected infected sites sent at admittance was included in the present study. All the jurisdiction was made by the attending physicians. Septic patients were followed up to day-90 or till decease. The protocol was approved by the ethics committees of Zhongda Hospital, School of Medicine, Southeast University (2015ZDSYLL044.0), with informed consent obtained from the patients or their representatives.

\section{Variables and definitions}

In the analysis of factors associated with positive culture, we selected a constellation of priori clinical variables known or expected to be potentially confounders involving demographic information including age, gender and bodyweight; clinical characteristics including comorbidities, patient source, priori ICU stay days and antibiotics exposure; vital signs; severity of sepsis by score evaluation including Sequential Organ Function Assessment (SOFA) score and Acute Physiology and Chronic Health Evaluation II (APACHE II) score; infection types and sites; laboratory findings involving inflammatory biomarkers including white blood cell count, C-reactive protein and procalcitonin, biomarkers reflecting organ functions including $\mathrm{PaO} 2$ to $\mathrm{FiO} 2$ ratio, serum lactate, hematocrit, platelet count, prothrombin time, activated partial thromboplastin time, D-dimer, serum creatinine, alanine aminotransferase, aspartate aminotransferase and troponin $\mathrm{l}$; and initial management of at admissions including vasopressor and mechanical ventilation. Sepsis was defined as suspected or confirmed infection with two or more criteria of the systemic inflammatory response; while severe sepsis was defined as sepsis accompanied with the 
presence of at least one life-threatening sepsis-related organ dysfunction ${ }^{[10,11]}$. The decision of the positive culture, negative culture or the contamination of the isolated pathogens remained to the attending physicians, and the appropriate antibiotics use was also by the judgment of the attending physicians. Appropriateness use of antibiotics was at the discretion of the attending

\section{Statistical Analysis}

Quantitative data were presented as mean \pm standard deviation (SD) or median (interquartile range, IQR) according to the data distributions. Categorial data were presented as proportions or ratios. Student $t$ test or one-way analysis of variance (ANOVA) was used for comparison of continuous variables in normal distribution, and Mann-Whitney Utest or Kruskal-Wallis $H$ test was used for comparison of quantitative variables with skewed distribution. Categorical data was compared using chi-square test or Fisher exact test, as appropriate. Univariable and multivariable logistic regression with forward LR methodology was used to determine the risk factors associated culture positivity, Univariable and multivariable odds ratios with $95 \%$ confidential intervals $(\mathrm{Cl})$ were calculated for potential association with culture positivity.

We created a propensity score using multivariable logistic regression to estimate the likelihood of positive culture of septic patients at admission to ICU. Propensity score matching based on the estimated probabilities were used to apply a one-to-one nearest neighbor matching algorithm between septic patients with positive culture and negative culture using a caliper width of 0.02 . Balance of covariates between patients with positive and negative cultures were assessed after matching using standardized differences.

Variables involved in the model to generate the propensity score included demographic information involving age and gender, baseline comorbidities, patient source, prior ICU stay days, prior antibiotics exposure, vital signs at admission, laboratory findings at admission, score evaluation at admission including APACHE II score and SOFA score, infection type, infection site and treatment at admission including mechanical ventilation and vasopressor use.

All statistical analyses were performed using IBM SPSS Statistics (ver. 23). A two-sided $P$-value less than 0.05 was considered as statistical significance.

\section{Results}

\section{Patient characteristics}

A total of 2322 patients were included in the CHESS study, and 403 patients were excluded with no microbial cultures taken at admission, with remaining 1919 patients finally involved in this study, and 962 (50.1\%) patients yielded a positive culture and 957 (49.9\%) with a negative culture.

Of all the patients enrolled in the present study, 1246 patients (64.9\%) were community-acquired infections, 640 (33.4\%) were hospital-acquired and the remaining 33 patients $(1.7 \%)$ were healthcarerelated. Lung was the leading site of infection (70.1\%), as followed by abdomen (25.2\%), urological 
(7.7\%) and bloodstream (5.0\%). A total of $71.7 \%$ of patients have samples obtained from the respiratory system, with a positive rate of $45.6 \%$ (622 in 1364 patients), $57.1 \%$ patients have samples taken from blood stream with a positive rate of $16.7 \%$ (183 in 1096 patients), and 19.7\% patients had samples from intra-abdomen cavity with positive rate of $45.9 \%$ (174 in 379 patients), $16.5 \%$ patients had samples from urological system yielding a positive rate of $20.6 \%$ (65 in 316 patients); and finally $1.9 \%$ patients with samples from central nervous system yielding a positive rate of $11.1 \%$ (4 in 36 patients).(Supplementary Table 1)

In patients with positive cultures, Gram-negative bacteria occupied $56.1 \%$ of the isolated micropathogen, with Acinetobacter baumannii (14.4\%), Klebsiella pneumoniae. (13.4\%) and Escherichia coli (12.2\%) as the most prevalent pathogens; while as for Gram-positive bacteria which took $19.8 \%$ positive results, Staphylococcus aureus (5.6\%), Enterococcus spp. (4.1\%). and Streptococcus viridans (2.7\%) was the most frequent pathogens obtained; Fungus were found in $13.6 \%$ of the positive cultures, with Candida albicans (6.3\%), Candida parapsilosis (1.4\%) and Aspergillus (1.2\%) most frequent pathogens isolated (Supplementary Table 2)

The characteristics of septic patients with positive and negative culture were summarized in Supplementary Table 3. Male was more likely to have a positive culture $(67.7 \%$ vs. $60.9 \%, P=0.002)$, and the patients with positive culture were of older age (year-olds, 65 [50 to 75] vs. 63 [48 to 74], $P=0.014$ ). Patients from emergency room ( $54.6 \%$ vs. $45.4 \%, P=0.017)$ and internal medicine $(54.5 \%$ vs. $45.5 \%, P=$ 0.018 ) seemed more likely to have negative culture results, while patients transferred from other hospitals $(13.6 \%$ vs. $9.9 \%, P=0.012)$ were more likely to have positive cultures. Patients underwent surgery, whether routine $(P=0.858)$ or emergency $(P=0.206)$, had no effects on the results of culture positivity. Patients with baseline comorbidities involving coronary heart disease $(19.1 \%$ vs. $15.4 \%, P=0.29)$ and solid tumor $(14.8 \%$ vs. $10.0 \%, P=0.002)$ had a higher percentage in positive culture results.

Patients with positive culture had a relatively prolonged priori ICU stay although the discrepancy was minimal (days, 1 [0 to 7] vs. 0 [0 to 5], $P=0.028$ ). Paradoxically, patients with prior exposure to antibiotics had a higher percentage with positive culture $(73.1 \%$ vs. $66.4 \%, P=0.001)$.

Infection type and site were associated with culture positivity, as patients with community-acquired infection prone to have more negative isolations $(70.4 \%$ vs. $59.5 \%, P<0.001)$, while hospital-acquired infection tended to have more positive culture results ( $38.9 \%$ vs. $27.8 \%, P<0.001)$. Patients with pneumonia and bloodstream infection were more likely to have positive culture results.

Culture positivity was significantly associated with sepsis severity, reflected as worse vital signs including higher body temperature (degree, 38.1 [36.8 to 38.6] vs. 37.9 [36.8 to 38.6], $P=0.013$ ) and heart rate (beats per minute, 115 [100 to 131] vs. 112 [97 to 129.5], $P=0.007$ ), also as higher APACHE II score (18 [13 to 24] vs. 16 [11 to 22], $P<0.001$ ) and SOFA score ( 8 [5 to 11] vs. 7 [ 4 to 10 ], $P=0.002$ ).

Laboratory tests found little relations between biomarkers reflecting organ function and blood culture results, and it was only that procalcitonin was higher in patients with positive culture $(\mathrm{ng} / \mathrm{mL}, 2.61[0.59$ 
to 12.9$]$ vs. 2.01 [0.50 to 9.41$], P=0.046$ )

No differences in mortality could be observed in the crude results between the patients with positive culture and those with negative culture, although the mortality in patients with positive culture was slightly higher ( $18.8 \%$ vs. $17.2 \%, P=0.165)$.

\section{Factors associated with culture positivity}

We subsequently included aforementioned factors potentially affected the culture positivity in a multivariable logistic regression model and found that male gender (OR 1.298, 95\% $\mathrm{Cl} 1.012$ to $1.666, P=$ 0.040), APACHE II score (OR 1.033, 95\% Cl 1.017 to 1.050, $P<0.001$ ), procalcitonin (OR $1.006,95 \% \mathrm{Cl}$ 1.002 to $1.010, P<0.001$ ), hospital-acquired infection (OR $1.878,95 \% \mathrm{Cl} 1.456$ to $2.423, P<0.001$ ) and bloodstream infection (OR $3.875,95 \% \mathrm{Cl} 2.023$ to $7.423, P<0.001$ ) as independent risk factors that were associated with positive culture in septic patients (Table 1).

\section{Propensity Score Match}

A total of 618 patients (309 patients with positive culture and 309 patients with negative culture) were finally enrolled after propensity score match, and no statistical significance were observed between the baseline demographic information, patient sources, comorbidities, prior ICU stay and prior antibiotics exposure, vital signs and laboratory findings at admission, score evaluation at admission including APACHE II score and SOFA score, infection type and treatment at admission. We found a significant decrease in patients with positive culture compared with those with negative culture $(38.2 \%$ vs. $48.2 \%, P$ $=0.012$ ) after matching (Supplementary Table 3)

A higher proportion of patients with targeted antibiotic treatment could be observed both before $(41.5 \%$ vs. $13.5 \%, P<0.001)$ and after PSM $(46.0 \%$ vs. $18.8 \%, P<0.001)$. We found a significant decrease in mortality after PSM (37.0\% vs. $46.2 \%, P=0.031)$, which was not observed before PSM (36.6\% vs. $35.8 \%$, $P=0.759)$ (Fig. 1).

\section{Discussion}

In this sub-analysis of a prospective observational cohort of septic patients, we found that septic patients with positive culture was associated with demographic characteristics, comorbidities, patient source, infection type and clinical features of the patients. PSM revealed that positive culture was associated with a significant decrease in mortality.

Patient characteristics including older age and male gender seemed to associated with positive culture, as was also indicated in previous studies ${ }^{[6,7]}$. The reason for this tendency was unclear, we thought this could partially contributed to that patients with older age usually had more severe sepsis, while septic patients more severe was likely to have positive cultures, as found in the present study. However, the association between male gender and culture positive remained to be identified. 
Patients from emergency medicine or internal medicine both had a tendency of negative cultures, while patients transferred from other hospitals were seemed to associate with positive cultures, as similar findings were also revealed in the previous studies ${ }^{[6,7,12]}$. This was consistent with the spectrum of the isolated pathogens, which was predominated by Gram-negative bacteria, usually isolated from hospitalacquired infections.

Comorbidities could affect the results of cultures, while the influence remained inconsistent. In the present study, we found that coronary heart failure and solid tumor were associated with culture positive. Previous study had found that diabetes mellitus may possibly related to a positive culture ${ }^{[6]}$. We extrapolated that some chronic comorbidities were more susceptible to have a micro-environment to have a positive culture, which could be related to a compromised immune system.

We found a higher proportion of patients with a positive culture who had priori exposure to anti-biotics. We extrapolated this could mostly due to the investigated populations, with more than half of the patients were hospital-acquired infections, and was very likely to have priori anti-biotics exposures. In a previous study, recent antibiotic exposure was found to be associated with increased hospital mortality in Gramnegative bacteremia infection with severe sepsis or septic shock, which was partly consistent with our findings ${ }^{[13]}$.

The septic patients with positive culture seemed to be more severe compared with negative ones, as APACHE II and SOFA score was significantly higher in culture-positive patients. This was probably could be explained by that the patients with positive results was related with more bacteria load and was more likely to be isolated.

Previous studies had revealed that culture-positive was associated with organ dysfunctions, so in this study, we also included laboratory findings at admittance, aimed to find some laboratory parameters that could be potentially associated with positive culture. Unfortunately, we found no connections between positive culture and laboratory parameters that reflected the organ function. We found from the multivariable regression, the only factor we've found as significantly related to culture positive was procalcitonin, as consistent with previous studies, that septic patients had a significantly elevated serum level of procalcitonin, indicating that procalcitonin might be a predictor ${ }^{[14]}$.

In a previous study, the authors introduced machine learning methodology in prediction of the culture positivity in suspected bloodstream infections ${ }^{[15]}$. However, in the in their study, the authors found the variables that has irrelevant to the clinical meanings ${ }^{[16]}$, and in the present study, we found some risk factor that could associated with culture positive in septic patients.

The mortality between culture positive and negative patients remained controversial in previous studies ${ }^{[2,}$ $6-8,12,17,18]$. in the present study, we found a higher mortality in patients with positive culture compared with negative cultures, from the crude results, however with no statistical significance ( $37.5 \% \mathrm{vs}$. $34.5 \%, P$ $=0.165$ ). We subsequently performed a PSM, and interestingly, we found a decrease in mortality after we 
matching the demographic information, source, infection type and severity of the septic patients (38.2\% vs. $48.2 \%, P=0.012)$. We noted a higher proportion of patients with complete resolve ( $15.9 \%$ vs. $10.4 \%)$. In the previous study, the authors found that patients with positive culture more likely to receive appropriate antibiotics treatment ${ }^{[12,19]}$, and this might partially explain the higher percentage of patients with complete resolve and decrease in mortality in positive culture. As in our study, we also noticed a higher proportion of patients with targeted antibiotic treatment, both before and after PSM. We further compared the mortality between septic patients between targeted and empirical antibiotics treatment, and interestingly, we found a significant decrease in mortality after PSM (37.0\% vs. $46.2 \%, P=0.031)$, which was not observed before PSM $(36.6 \%$ vs. $35.8 \%, P=0.759)$. We extrapolated the appropriate use of antibiotics might be one of the reasons that contributed to the decreased in mortality in culture positive patients, while in the crude results, the effect of appropriate antibiotic use was masked by the cofounding factors.

We thought the factors associated with culture positive in septic patients were neither very strong predictors, nor could be manipulated in the clinical practice. On the other side, technical improvement ${ }^{[20,}$ 21], multiple sending of the samples definite affect culture positive, as culture positive was very likely to be associated with decrease in mortality, we thought it would be much encouraged for a given septic patients to obtain positive culture whenever possible.

Our study had some limitations, we did not include the difference in antibiotics administration between patients with positive and negative cultures. We assumed that the discrepancies in mortality in two groups of patients was contributed to the targeted antibiotics management which was guided by the results of the positive culture, which needed further investigations. We found no factors that could intervened for to improve the culture positive.

\section{Conclusion}

The factors associated with culture positive remained to be further identified, although patients' demographic characteristics, infection type and the severity of sepsis may affect the results of culture. In spite of the higher mortality in septic patients with positive culture, PSM revealed that positive culture was associated with a decrease mortality for a given septic patients, suggesting that patients might be benefitted from positive culture and obtaining positive culture as possible should be strongly encouraged.

\section{Abbreviations}

ANOVA one-way analysis of variance, APACHE II Acute Physiology and Chronic Health Evaluation II, CHESS Chinese Epidemiological Study of Sepsis, Cl confidential interval, ICU intensive care unit, IQR interquartile range, OR odds ratio, PSM propensity score matching, SD standard deviation, SOFA Sequential Organ Function Assessment

\section{Declarations}




\section{Ethics approval and consent to participate}

This study was performed in accordance with the Declaration of Helsinki and approved by Research Ethics Board of Zhongda Hospital (Southeast University, Nanjing, China; 2015ZDSYLL042.0). Written informed consent was obtained by close relatives of the participating patients.

\section{Consent for publication}

Not applicable.

\section{Availability of data and materials}

The datasets used and/or analysed during the study available from the corresponding author on reasonable request.

\section{Competing interests}

The authors declare that they have no competing interests.

\section{Funding}

This work was partially supported by Jiangsu Province's Key Discipline / Laboratory of Medicine (ZDXKA2016025), Jiangsu Provincial Key Research and Development Program (SBE2018750279) and Fundamental Scientific Research Foundation of Southeast University (2242020K40167)

\section{Authors' contributions}

W-C, JF-X carried out the analysis and interpretation of data and participated in drafting, editing and submitting the manuscript. Q-S, F-P, Q-L, M-X and Y-Z participated in recording and computerizing the data of the patients and the patients' treatments. HB-Q and $Y-Y$ was responsible for conception, design and coordination of the study, and revising the manuscript for important intellectual content. All authors read and approved the final manuscript.

\section{Acknowledgements}

Not applicable.

\section{References}

1. Singer M, Deutschman CS, Seymour CW, et al. The Third International Consensus Definitions for Sepsis and Septic Shock (Sepsis-3). JAMA. 2016;315(8):801-10. 
2. Levy MM, Artigas A, Phillips GS, et al. Outcomes of the Surviving Sepsis Campaign in intensive care units in the USA and Europe: a prospective cohort study. Lancet Infect Dis. 2012;12(12):919-24.

3. Fleischmann C, Scherag A, Adhikari NK, et al. Assessment of Global Incidence and Mortality of Hospital-treated Sepsis. Current Estimates and Limitations. Am J Respir Crit Care Med. 2016;193(3):259-72.

4. Rhodes A, Evans LE, Alhazzani W, et al. Surviving Sepsis Campaign: International Guidelines for Management of Sepsis and Septic Shock: 2016. Intensive Care Med. 2017;43(3):304-77.

5. Tabah A, Bassetti M, Kollef MH, et al. Antimicrobial de-escalation in critically ill patients: a position statement from a task force of the European Society of Intensive Care Medicine (ESICM) and European Society of Clinical Microbiology and Infectious Diseases (ESCMID) Critically III Patients Study Group (ESGCIP). Intensive Care Med. 2020;46(2):245-65.

6. Phua J, Ngerng W, See K, et al. Characteristics and outcomes of culture-negative versus culturepositive severe sepsis. Crit Care. 2013;17(5):R202.

7. Nannan Panday RS, Lammers EMJ, Alam N, et al. An overview of positive cultures and clinical outcomes in septic patients: a sub-analysis of the Prehospital Antibiotics Against Sepsis (PHANTASi) trial. Crit Care. 2019;23(1):182.

8. Gupta S, Sakhuja A, Kumar G, et al. Culture-Negative Severe Sepsis: Nationwide Trends and Outcomes. Chest. 2016;150(6):1251-9.

9. Xie J, Wang H, Kang Y, et al. The Epidemiology of Sepsis in Chinese ICUs: A National Cross-Sectional Survey. Crit Care Med. 2020;48(3):e209-e18.

10. American College of Chest Physicians/Society of Critical Care Medicine Consensus Conference: definitions for sepsis and organ failure and guidelines for the use of innovative therapies in sepsis. Crit Care Med. 1992;20(6):864 - 74.

11. Dellinger RP, Levy MM, Rhodes A, et al. Surviving Sepsis Campaign: international guidelines for management of severe sepsis and septic shock, 2012. Intensive Care Med. 2013;39(2):165-228.

12. Kethireddy S, Bilgili B, Sees A, et al. Culture-Negative Septic Shock Compared With Culture-Positive Septic Shock: A Retrospective Cohort Study. Crit Care Med. 2018;46(4):506-12.

13. Johnson MT, Reichley R, Hoppe-Bauer J, et al. Impact of previous antibiotic therapy on outcome of Gram-negative severe sepsis. Crit Care Med. 2011;39(8):1859-65.

14. Muller F, Christ-Crain M, Bregenzer T, et al. Procalcitonin levels predict bacteremia in patients with community-acquired pneumonia: a prospective cohort trial. Chest. 2010;138(1):121-9.

15. Roimi M, Neuberger $A$, Shrot $A$, et al. Early diagnosis of bloodstream infections in the intensive care unit using machine-learning algorithms. Intensive Care Med. 2020;46(3):454-62.

16. Liu VX, Wiens J. 'No growth to date'? Predicting positive blood cultures in critical illness. Intensive Care Med. 2020;46(3):525-7.

17. Brun-Buisson C, Doyon F, Carlet J, et al. Incidence, risk factors, and outcome of severe sepsis and septic shock in adults. A multicenter prospective study in intensive care units. French ICU Group for 
Severe Sepsis. JAMA. 1995;274(12):968-74.

18. Rangel-Frausto MS, Pittet $D$, Costigan $M$, et al. The natural history of the systemic inflammatory response syndrome (SIRS). A prospective study. JAMA. 1995;273(2):117-23.

19. Harbarth S, Garbino J, Pugin J, et al. Inappropriate initial antimicrobial therapy and its effect on survival in a clinical trial of immunomodulating therapy for severe sepsis. Am J Med. 2003;115(7):529-35.

20. Bloos F, Hinder F, Becker $\mathrm{K}$, et al. A multicenter trial to compare blood culture with polymerase chain reaction in severe human sepsis. Intensive Care Med. 2010;36(2):241-7.

21. Yanagihara $\mathrm{K}$, Kitagawa $\mathrm{Y}$, Tomonaga $\mathrm{M}$, et al. Evaluation of pathogen detection from clinical samples by real-time polymerase chain reaction using a sepsis pathogen DNA detection kit. Crit Care. 2010;14(4):R159.

\section{Tables}

Table 1 Factors Associated with Positive Culture in Septic Patients by Univariate and Multivariate logistic regression 


\begin{tabular}{|c|c|c|c|c|}
\hline & Univariate & & Multivariate & \\
\hline Variables & OR $(95 \% \mathrm{Cl})$ & $\begin{array}{l}P- \\
\text { value }\end{array}$ & OR $(95 \% \mathrm{Cl})$ & $\begin{array}{l}P \text { - } \\
\text { value }\end{array}$ \\
\hline Age, per year & $\begin{array}{l}1.006 \text { (1.001 to } \\
1.012)\end{array}$ & 0.011 & & \\
\hline Male & $\begin{array}{l}1.343(1.113 \text { to } \\
1.620)\end{array}$ & 0.002 & $\begin{array}{l}1.298(1.012 \text { to } \\
1.666)\end{array}$ & 0.040 \\
\hline Patients from emergency room & $\begin{array}{l}0.778(0.632 \text { to } \\
0.957)\end{array}$ & 0.017 & & \\
\hline Patients from internal medicine & $\begin{array}{l}0.780 \text { ( } 0.635 \text { to } \\
0.958)\end{array}$ & 0.018 & & \\
\hline Patients transferred & $\begin{array}{l}1.430(1.080 \text { to } \\
1.894)\end{array}$ & 0.012 & & \\
\hline $\begin{array}{l}\text { Patients with coronary heart } \\
\text { disease }\end{array}$ & $\begin{array}{l}1.303(1.027 \text { to } \\
1.654)\end{array}$ & 0.029 & & \\
\hline Patients with solid tumor & $\begin{array}{l}1.553 \text { ( } 1.178 \text { to } \\
2.047)\end{array}$ & 0.002 & & \\
\hline Prior ICU stay & $\begin{array}{l}1.004 \text { (0.999 to } \\
1.009)\end{array}$ & 0.131 & & \\
\hline Prior antibiotics exposure & $\begin{array}{l}1.376(1.132 \text { to } \\
1.674)\end{array}$ & 0.001 & & \\
\hline Mechanical ventilation & $\begin{array}{l}1.368 \text { (1.092 to } \\
1.713)\end{array}$ & 0.006 & & \\
\hline Body temperature & $\begin{array}{l}1.092 \text { (1.018 to } \\
1.172)\end{array}$ & 0.014 & & \\
\hline Heart rate & $\begin{array}{l}1.004 \text { (1.001 to } \\
1.009)\end{array}$ & 0.024 & & \\
\hline Procalcitonin & $\begin{array}{l}1.006 \text { (1.002 to } \\
1.010)\end{array}$ & 0.005 & $\begin{array}{l}1.006 \text { (1.002 to } \\
1.010)\end{array}$ & $\begin{array}{l}< \\
0.001\end{array}$ \\
\hline APACHE II score & $\begin{array}{l}1.026(1.015 \text { to } \\
1.038)\end{array}$ & <. 001 & $\begin{array}{l}1.033(1.017 \text { to } \\
1.050)\end{array}$ & $<.001$ \\
\hline SOFA score & $\begin{array}{l}1.036(1.014 \text { to } \\
1.059)\end{array}$ & 0.002 & & \\
\hline Community acquired infection & $\begin{array}{l}0.616(0.510 \text { to } \\
0.744)\end{array}$ & < 0.001 & & \\
\hline Hospital acquired infection & $\begin{array}{l}1.652(1.364 \text { to } \\
2.002)\end{array}$ & <. 001 & $\begin{array}{l}1.878 \text { ( } 1.456 \text { to } \\
2.423)\end{array}$ & $\begin{array}{l}< \\
0.001\end{array}$ \\
\hline Pneumonia & $\begin{array}{l}1.372(1.128 \text { to } \\
1.670)\end{array}$ & 0.002 & & \\
\hline
\end{tabular}


$<$

\section{Figures}

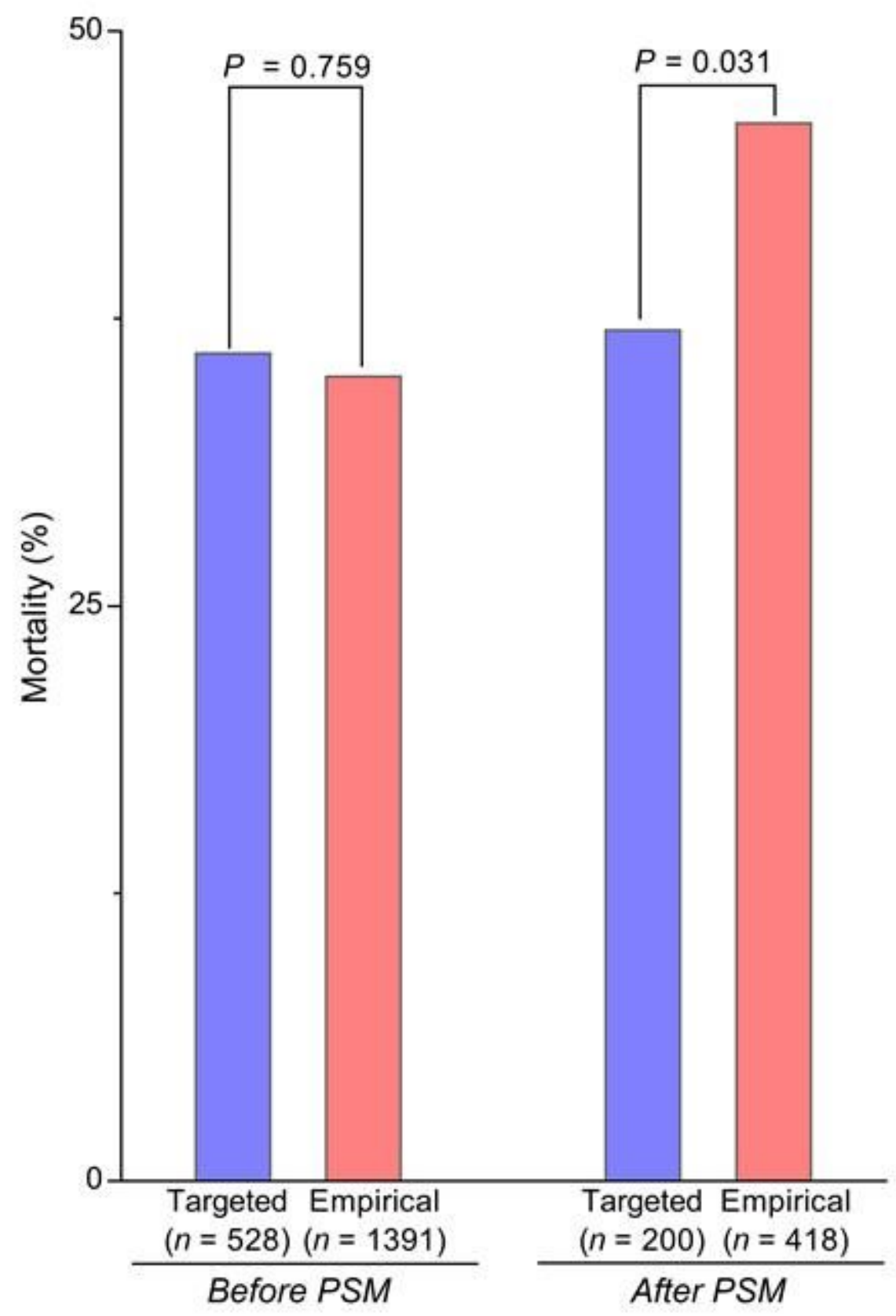

Figure 1

Mortality in Empirical and Targeted Use of Antibiotics Before and After Propensity Score Matching

\section{Supplementary Files}


This is a list of supplementary files associated with this preprint. Click to download.

- SupplementaryTable3.docx

- SupplementaryTable2.docx

- SupplementaryTable1.docx 